\title{
Attitude Stability Analysis for Stick-Slip-Induced Disturbances of Extended Structure with Tension Control Mechanism *
}

\author{
Shigemune TANIWAKI**, Yasushi KOJIMA*** and Yoshiaki OHKAMI**** \\ ${ }^{* *}$ Graduate School of Science and Engineering, Ehime University \\ 3 Bunkyo-cho, Matsuyama 790-8577, Japan \\ E-mail: staniwaki@eng.ehime-u.ac.jp \\ ***Japan Aerospace Exploration Agency \\ Tsukuba Space Center, 2-1-1 Sengen, Tsukuba 305-8505, Japan \\ ****Keio University \\ 2-15-45 Mita, Minato-ku, Tokyo 108-8345, Japan
}

\begin{abstract}
An analytical approach is developed for determining the spacecraft attitude response to disturbances caused by the stick-slip motion of a large extended structure with a tension control mechanism. In this method, attitude dynamics with a flexible appendage is formulated as a general constrained mode model, and coupling with the stick-slip motion is formulated as the transition of equilibrium states of modal parameters. Numerical calculations are presented for the flexible solar array on the Advanced Earth Observing Satellite. This satellite experienced unexpected attitude oscillations due to the stick-slip motion while operating in orbit.
\end{abstract}

Key words: Spacecraft, Attitude Control, Stick-Slip, Self-Excited Oscillation

\section{Nomenclature}

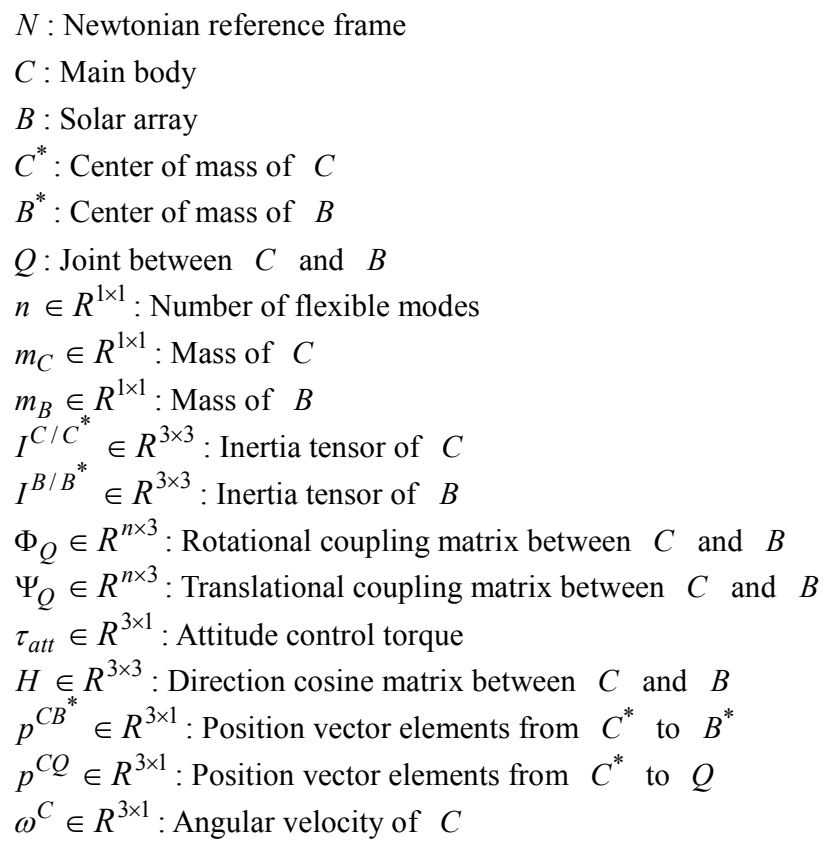


$v^{C^{*}} \in R^{3 \times 1}:$ Translational velocity of $C^{*}$

$\omega_{i} \in R^{1 \times 1}:$ Angular frequency of $i$ th flexible mode $(i=1, \ldots, n)$

$\varsigma_{i} \in R^{1 \times 1}$ : Damping ratio of $i$ th flexible mode $(i=1, \ldots, n)$

$q_{\text {att }} \in R^{3 \times 1}$ : Attitude angle of $C$

$q_{\text {dis }} \in R^{3 \times 1}:$ Displacement of $C^{*}$

$q_{f l x} \in R^{n \times 1}:$ Displacement of flexible modes

$O_{i j} \in R^{i \times j}:$ Zero matrix of dimension $i \times j$

$U_{i i} \in R^{i \times i}$ : Unit matrix of dimension $i \times i$

\section{Introduction}

The Advanced Earth Observing Satellite (ADEOS), which was launched into orbit on August 17, 1996, has a 23-m-long solar array, as shown in Fig. 1. The structure of this solar array is shown in Fig. 2. It consists of an extension mast, a blanket, a tension control mechanism (TCM), and a boom. The extension mast has a truss structure. The blanket has a flexible membrane structure on which solar-electric power generation cells are mounted. The blanket does not have enough stiffness by itself, but both ends of the blanket are pulled out by the

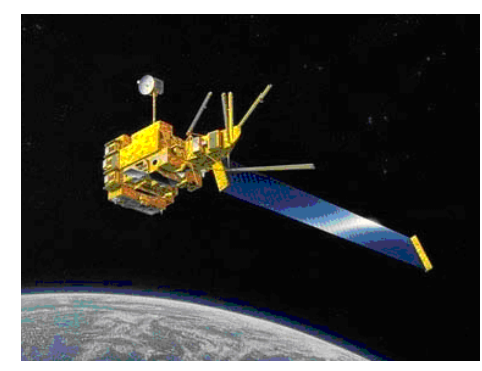

Fig. 1 ADEOS in orbit

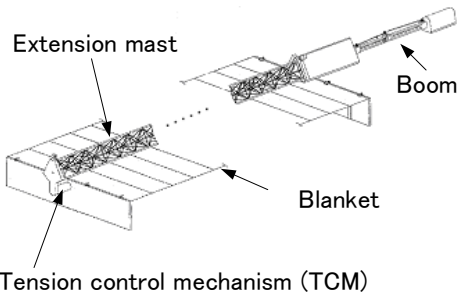

Fig. 2 Structure of the solar array TCM fixed on the extension mast, and, overall, the whole solar array has enough stiffness. This type of solar array is called a flexible solar array, whereas a solar array with solar-electric power generation cells placed directly on a relatively rigid plate is called a rigid solar array. If the length of the solar array exceeds $20 \mathrm{~m}$, the flexible solar array is superior to the rigid one in terms of saving weight and being easy to store within the fairing of the launch vehicle. Recent space missions have tended to require much more power than before, and the flexible solar array is regarded as a promising solution to this need. The decision to use a flexible solar array on the ADEOS for the first time was made by the National Space Development Agency of Japan (NASDA) considering the good prospects for the future. However, during normal operating modes in orbit, abnormal attitude jitter was observed in the telemetry data. Extensive analysis was carried out by studying the telemetry data and performing ground experiments, and it turned out that the TCM caused a stick-slip phenomenon, which is a kind of self-excited oscillation. Therefore, when the successor satellite ADEOS-II was developed, the design of the TCM was improved. ADEOS-II was launched into orbit on December 14, 2002. During its operation, the TCM worked well and the stick-slip phenomenon did not occur.

A similar phenomenon to the one that affected ADEOS also occurred on the Hubble Space Telescope (HST). The solar array of the HST also has a structure where the flexible membrane is pulled out by the tension mechanism and stiffness is maintained. Through the process of finding out the problems with the HST in particular, and through research on similar phenomena, various kinds of numerical models for coping with a solar array with a tension mechanism were developed ${ }^{(1)-(6)}$. Such models are useful for investigating the complex dynamics of the solar array, but their effects on attitude stability have not been directly analyzed. Therefore, in our study, we developed an attitude dynamics model combined with the solar array model. In this method, attitude dynamics with the flexible appendage is formulated as a general constrained mode model, and coupling with the TCM stick-slip motion is formulated as the transition of the equilibrium states of modal parameters. Our model is useful not only for the time domain analysis of attitude dynamics but also for a frequency domain analysis. Numerical calculations are presented for ADEOS, and the attitude jitter found in the telemetry data is reproduced. Our method was also 
applied to ADEOS-II to investigate the attitude stability against stick-slip motion, and its robustness was verified.

This paper is organized as follows. Section 2 overviews the TCM configuration of ADEOS and the stick-slip phenomenon. Section 3 formulates the attitude dynamics with the stick-slip mechanism. Section 4 presents numerical simulation results for ADEOS. Section 5 discusses how to improve robustness against stick-slip motion and presents simulation results for ADEOS-II. Section 6 summarizes the main achievements of this paper.

\section{TCM Configuration and Stick-Slip Phenomenon of ADEOS}

\subsection{TCM Configuration}

The configuration of the solar array on ADEOS is shown in Fig. 3. When the thermal condition varies, both the blanket and the extension mast cause distortion. But their coefficients of thermal expansion are different and their time constants for reacting to changes in thermal conditions are also different. As a result, their distortion lengths are

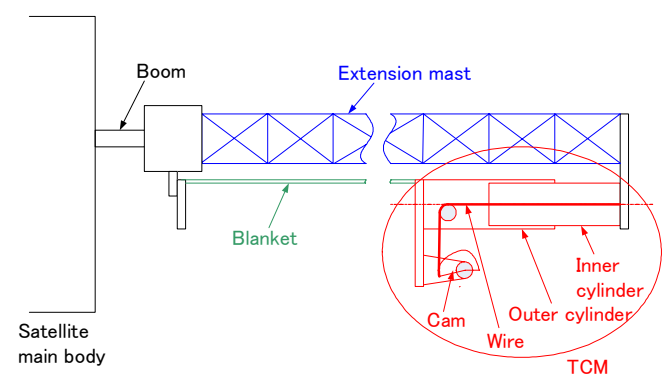

Fig. 3 TCM configuration different. The TCM absorbs this relative distortion length and also keeps the tension of the blanket constant. The direction of the tension is kept parallel to the longitudinal direction of the solar array by inner and outer cylinders. Depending on the relative distortion length, a cam with a torsion spring reels the wire in or out, and the relative position between the inner and outer cylinders is passively adjusted. The radius of the cam is designed to generate constant tension for an arbitrary rotation angle. For example, if the length of the extension mast exceeds that of the blanket, the inner cylinder is extracted from the outer cylinder and the cam reels out the wire.

\subsection{Stick-Slip}

Stick-slip is a kind of self-excited oscillation where a system acted upon by friction force, elastic force, and inertia force becomes instable. In general, it happens when friction decreases as slip velocity increases.

Abnormal attitude jitter in ADEOS was observed in particular right after the satellite moved from being in sunlight to being eclipsed, and vice versa. Extensive analysis was carried out by studying the telemetry data and performing ground experiments, and it turned out that the TCM caused the stick-slip motion between the inner cylinder and the outer cylinder when the thermal condition changed. This mechanism is summarized as follows.

When the inner and outer cylinders are stuck, the TCM never absorbs the relative distortion and never keeps the constant tension. At this time, thermal distortion is absorbed by elastic deformation of the blanket and the extension mast. The greater the thermal distortion is, the greater the elastic deformation and the elastic force are. When the force acting on the sticking portion exceeds the sticking strength between the inner and outer cylinders, slip motion occurs and the elastic energy is released at that moment. This causes abnormal vibration in the flexible modes of the solar array and abnormal jitter in the attitude. When the relative velocity between the inner and outer cylinders reduces, they become stuck again. Such a phenomenon is repeated and self-excited oscillation or stick-slip motion occurs. This continues until the thermal distortion converges.

\section{Dynamics Model}

\subsection{Attitude Dynamics Model}

When the stick-slip motion coupled with an attitude motion is formulated, a normal 
attitude dynamics model is expanded to include the effects of the stick slip motion. In this section, first, a normal attitude dynamics model is formulated. In general, the attitude motion of a main body is coupled with the translational motion of the main body and the flexible motion of the flexible solar array. When the attitude angle is small, approximate equations $\dot{q}_{a t t} \approx \omega^{C}$ and $\dot{q}_{d i s} \approx v^{C^{*}}$ exist and the dynamics model is formulated as follows.

$$
M \ddot{q}+D \dot{q}+K q=F \tau_{\text {att }},
$$

where

$$
\begin{aligned}
& q=\left[\begin{array}{l}
q_{a t t} \\
q_{d i s} \\
q_{f l x}
\end{array}\right] \in R^{(6+n) \times 1}, M=\left[\begin{array}{lll}
A_{11} & A_{12} & A_{13} \\
A_{21} & A_{22} & A_{23} \\
A_{31} & A_{32} & A_{33}
\end{array}\right] \in R^{(6+n) \times(6+n)}, \\
& D=\left[\begin{array}{lll}
O_{33} & O_{33} & O_{3 n} \\
O_{33} & O_{33} & O_{3 n} \\
O_{n 3} & O_{n 3} & D_{f l x}
\end{array}\right] \in R^{(6+n) \times(6+n)}, K=\left[\begin{array}{lll}
O_{33} & O_{33} & O_{3 n} \\
O_{33} & O_{33} & O_{3 n} \\
O_{n 3} & O_{n 3} & K_{f l x}
\end{array}\right] \in R^{(6+n) \times(6+n)}, \\
& F=\left[\begin{array}{l}
U_{33} \\
O_{33} \\
O_{n 3}
\end{array}\right] \in R^{(6+n) \times 3}, \\
& A_{11}=I^{C / C^{*}}+H^{T} I^{B / B^{*}} H+m_{B} \widetilde{p}^{C B^{* T}} \widetilde{p}^{C B^{*}} \in R^{3 \times 3}, \\
& A_{12}=A_{21}^{T}=-m_{B} \widetilde{p}^{C B^{* T}} \in R^{3 \times 3}, A_{13}=A_{31}^{T}=H^{T} \Phi_{Q}^{T}-\widetilde{p}^{C Q^{T}} H^{T} \Psi_{Q}^{T} \in R^{3 \times n}, \\
& A_{22}=\left(m_{C}+m_{B}\right) U_{33} \in R^{3 \times 3}, A_{23}=A_{32}^{T}=H^{T} \Psi_{Q}^{T} \in R^{3 \times n}, A_{33}=U_{n n} \in R^{n \times n}, \\
& D_{f l x}=\operatorname{diag}\left\{\cdots \quad 2 \varsigma_{i} \omega_{i} \quad \cdots\right\} \in R^{n \times n}, K_{f l x}=\operatorname{diag}\left\{\cdots \quad \omega_{i}^{2} \quad \cdots\right\} \in R^{n \times n}
\end{aligned}
$$

and a tilde operator $\widetilde{r}$ for an arbitrary vector $r=\left[\begin{array}{lll}r_{1} & r_{2} & r_{3}\end{array}\right]^{T} \in R^{3 \times 1}$ is defined as

$$
\widetilde{r}=\left[\begin{array}{ccc}
0 & -r_{3} & r_{2} \\
r_{3} & 0 & -r_{1} \\
-r_{2} & r_{1} & 0
\end{array}\right] \in R^{3 \times 3}
$$

Equation (1) is generally known as a constrained mode model where the rigid motion is represented by physical parameters $q_{a t t}$ and $q_{d i s}$ and the flexible motion is represented by modal parameters $q_{f f x}$. In general, an inertia matrix $M$ is not diagonal, and the attitude motion, translational motion, and structural vibration are coupled with each other. In particular, $A_{13}$ and $A_{31}$ represent the coupling effects between the attitude motion and the flexible motion. The effects of damping forces $D_{f l x} \dot{q}_{f l x}$ and elastic forces $K_{f l x} q_{f l x}$ of the solar array are formulated in the second and third terms of Eq. (1).

\subsection{Stick-Slip Model}

The dynamics equation of the solar array is represented in the third block line in Eq. (1). It can be rewritten as

$$
A_{33} \ddot{q}_{f l x}+D_{f f x} \dot{q}_{f l x}+K_{f l x} q_{f l x}+A_{31} \ddot{q}_{a t t}+A_{32} \ddot{q}_{d i s}=O_{n 1}
$$

Stick-slip motion occurs at the solar array. Therefore, Eq. (2) is modified to include the effects of the stick-slip motion. In general, modal displacements $q_{f f x}$ correspond to the deformation from the equilibrium condition. Modal displacements are caused by the elasticity of the solar array, but the center of the oscillations is around the equilibrium states 
when the elastic properties are not changed. But when the thermal condition of the solar array changes, the array causes deformation not because of the elastic property but because of the thermal strain. Such a distortion varies equilibrium states. Both the thermal strain and the elastic deformation apply stress to the structure of the solar array. When the inner and outer cylinders are stuck, such a stress also acts between the cylinders. When the stress exceeds the sticking strength, slip motion occurs.

Such a phenomenon can be illustrated by the simple $2 \mathrm{DOF}$ (two degrees of freedom) mass-spring-damper system shown in Fig. 4. The nominal equilibrium

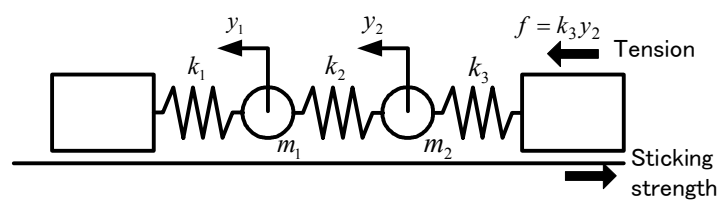

(a) nominal equilibrium states

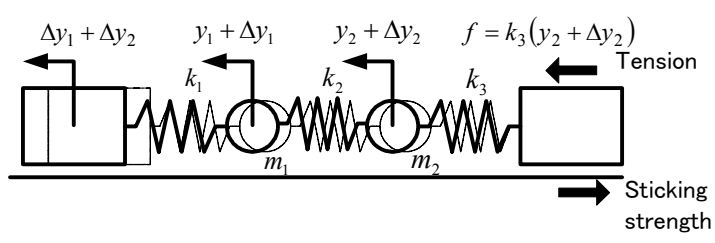

(b) transited equilibrium states

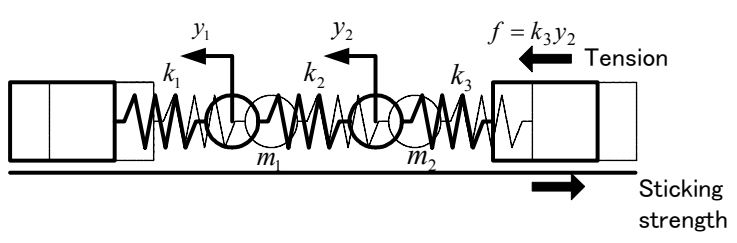

(c) equilibrium states right after the slip motion

Fig. 4 Thermal distortion and flexible deformation states mean the equilibrium states where thermal distortion does not occur, and the transited equilibrium states mean the equilibrium states where thermal distortion does occur. Displacements of masses $m_{1} \in R^{1 \times 1}$ and $m_{2} \in R^{1 \times 1}$ are denoted as $y_{1} \in R^{1 \times 1}$ and $y_{2} \in R^{1 \times 1}$, spring constants are denoted as $k_{1} \in R^{1 \times 1}$ and $k_{2} \in R^{1 \times 1}$, the transitions of the equilibrium states caused by the thermal distortion are denoted as $\Delta y_{1} \in R^{1 \times 1}$ and $\Delta y_{2} \in R^{1 \times 1}$, and the tension acting on the sticking point is denoted as $f \in R^{1 \times 1}$. The dynamics around the nominal equilibrium states corresponding to Fig. 4(a) is formulated

$$
\left[\begin{array}{cc}
m_{1} & 0 \\
0 & m_{2}
\end{array}\right]\left[\begin{array}{l}
\ddot{y}_{1} \\
\ddot{y}_{2}
\end{array}\right]+\left[\begin{array}{cc}
k_{1}+k_{2} & -k_{2} \\
-k_{2} & k_{2}+k_{3}
\end{array}\right]\left[\begin{array}{l}
y_{1} \\
y_{2}
\end{array}\right]=\left[\begin{array}{l}
0 \\
0
\end{array}\right] .
$$

When thermal distortion $\Delta y_{1}, \Delta y_{2}$ occurs, the dynamics around the transited equilibrium states corresponding to Fig. 4(b) is formulated

$$
\left[\begin{array}{cc}
m_{1} & 0 \\
0 & m_{2}
\end{array}\right]\left[\begin{array}{l}
\ddot{y}_{1} \\
\ddot{y}_{2}
\end{array}\right]+\left[\begin{array}{cc}
k_{1}+k_{2} & -k_{2} \\
-k_{2} & k_{2}+k_{3}
\end{array}\right]\left[\begin{array}{l}
y_{1} \\
y_{2}
\end{array}\right]=-\left[\begin{array}{cc}
k_{1}+k_{2} & -k_{2} \\
-k_{2} & k_{2}+k_{3}
\end{array}\right]\left[\begin{array}{l}
\Delta y_{1} \\
\Delta y_{2}
\end{array}\right] .
$$

In this formulation, it is noted that the thermal strain causes the structural deformation, but its variation is slower than the motion caused by the flexible deformation. Therefore, the structural deformation caused by the thermal strain is modeled as a variation of the equilibrium states of modal displacements, and the acceleration term of the thermal deformation is neglected.

At this time, the tension acting on the stick portion is formulated as

$$
f=k_{3}\left(y_{2}+\Delta y_{2}\right) \text {. }
$$

At the moment the tension exceeds the sticking strength, slip motion occurs. Then the tension is reduced and the slipping portion becomes stuck again. If the length of the slipping motion is assumed to be equal to $\Delta y_{1}+\Delta y_{2}$, the equilibrium states right after the slip motion shown in Fig. 4(c) are numerically equivalent to the nominal equilibrium states shown in Fig. 4(a), and they are formulated as Eq. (3).

Next, the stick-slip motion occurring in the solar array is formulated. When Eq. (3) takes into account damping forces that are proportional to modal displacement velocities, it 
has the same form as Eq. (2), but without the attitude coupling term $A_{31} \ddot{q}_{\text {att }}$ and the translational motion coupling term $A_{32} \ddot{q}_{\text {dis }}$. Therefore, Eq. (2) can be expanded as follows when the thermal distortion is considered, the same as in Eq. (4).

$$
A_{33} \ddot{q}_{f l x}+D_{f l x} \dot{q}_{f l x}+K_{f l x} q_{f l x}+A_{31} \ddot{q}_{a t t}=-K_{f l x} \Delta q_{f l x},
$$

where $\Delta q_{f l x} \in R^{n \times 1}$ represents the transition of the equilibrium states caused by thermal distortion. If the third block line in Eq. (1) is replaced by Eq. (6), we get the following equation.

$$
M \ddot{q}+D \dot{q}+K q=\bar{F} u
$$

where

$$
u=\left[\begin{array}{c}
\tau_{a t t} \\
-K_{f l x} \Delta q_{f l x}
\end{array}\right] \in R^{(3+n) \times 1}, \bar{F}=\left[\begin{array}{cc}
U_{33} & O_{3 n} \\
O_{33} & O_{3 n} \\
O_{n 3} & U_{n n}
\end{array}\right] \in R^{(6+n) \times(3+n)}
$$

In Eq. (7), $-K_{f l x} \Delta q_{f l x}$ is regarded as disturbance inputs to the flexible modes caused by the discharge of the elastic energy at the moment of the slip motion.

The tension acting on the TCM is formulated as follows, the same as for Eq. (5).

$$
f=\bar{K}_{f l x}\left(q_{f l x}+\Delta q_{f l x}\right)
$$

The tension acting on the TCM depends not only on the modal displacements but also on the modal shapes. Therefore, the timing of the slip motion is determined from these variables. Coefficient matrix $\bar{K}_{f l x} \in R^{1 \times n}$ in Eq. (8) is determined taking into account these effects. At the moment the tension exceeds the sticking strength, slip motion occurs and the equilibrium states go back to the nominal states. This assumption is reasonable because the telemetry data of ADEOS shows that the tension of the TCM went back to almost the normal value after slip motion. It was also verified through the telemetry data that the elapsed time from the beginning to the end of the slip motion was much shorter than the sticking period. Therefore, the slip motion is assumed to occur within an infinitesimal time.

The state transition of our model is shown in Fig. 5. While the tension calculated with Eq. (8) is less than the sticking strength, the sticking condition is maintained. At the moment the tension exceeds the sticking strength, slip motion occurs and the equilibrium states go back to the nominal states. This phenomenon is repeated during the thermal distortion.

\section{Numerical Simulation}

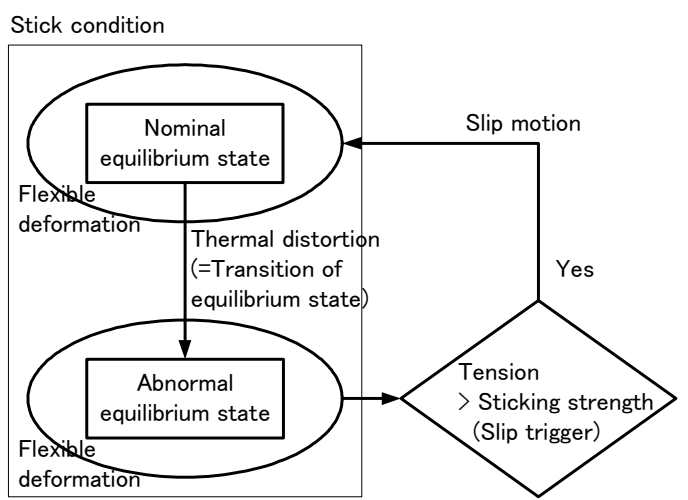

Fig.5 State transition

\subsection{Dynamics Analysis}

The dynamics model formulated as Eq. (7), the tension model formulated as Eq. (8), and the state transition model represented as Fig. 5 were used in numerical simulations. Simulation results for ADEOS in sunlight are shown in Fig. 6. In this analysis, the thermal strain $\Delta q_{f l x}$ was calculated from the blanket temperature, the extension mast temperature, and their physical parameters, where the temperature data was obtained from the telemetry data. Then, $\Delta q_{f l x}$ was substituted into Eq. (7) and the dynamics model was solved. The thermal distortion, equilibrium states, and modal displacement are shown in Fig. 6(a). Only the first modal displacement is shown because its effect on the distortion is dominant. Even 
when the blanket is exposed to the sun, the extension mast is located behind the blanket, and the time constant of the thermal distortion of the extension mast is larger than that of the blanket. Therefore, once the transitional thermal expansion of the blanket has settled down right after the satellite leaves an eclipsed region and enters the sunlight, the thermal expansion ratio of the extension mast is larger than that of the blanket, and the inner cylinder of the TCM is extracted from the outer cylinder. As shown in Fig. 6(a), the time cycle of the stick-slip is about $150 \mathrm{~s}$, and the modal displacement includes not only frequency elements of its mode, but also elements synchronized with the stick-slip cycle. Figure 6(b) shows the attitude angular velocity for each axis. It also includes not only the frequency elements of the first mode, but also elements synchronized with the stick-slip cycle.

The telemetry data for attitude angular velocity in the sunlight is shown in Fig. 7. It includes frequency elements closing the first flexible mode. It also includes frequency elements whose time cycle is from $90 \mathrm{~s}$ to $120 \mathrm{~s}$. Figure 6(b) also includes frequency elements whose time cycle is about $150 \mathrm{~s}$. Therefore, both the telemetry data and the simulation results showed the same tendency. Strictly speaking, the simulated stick-slip cycles include a $50 \%$ error because of the uncertainty of the sticking strength, uncertainty of the damping ratio of the first mode, and so on.

Simulation results for eclipsed regions are shown in Fig. 8. When the satellite leaves the sunlight and enters an eclipsed region, both the extension mast and the blanket are constricted, but the blanket is constricted much more quickly. As a result, the inner cylinder of the TCM is extracted from the outer cylinder. In Fig 8(a), the magnitude of the thermal distortion velocity was large right after the satellite left the sunlight, but it becomes smaller and smaller as time progresses. Therefore, the time cycle of the stick-slip is short at the beginning but became longer and longer; that is, it varied from $10 \mathrm{~s}$ to $40 \mathrm{~s}$. The attitude angular velocity is shown in Fig. 8(b). The effects of the first flexible mode were dominant. When the frequency of the stick-slip motion was close to that of the first flexible mode, the amplitude of the attitude angular velocity tended to be large. The distance between the modal frequency and the stick-slip frequency was not periodic in the case of an eclipsed region, and then the amplitude of the angular velocity changed irregularly.

Telemetry data for attitude angular velocity in an eclipsed region is shown in Fig. 9. Though Fig. 9 also shows irregular variations in the amplitude, meaning that our method is useful to verify the tendency of the attitude stability against stick-slip motion, Fig. 9 shows a much larger variation in amplitude than Fig. 8(b). Such a difference is caused by uncertainties in the simulation parameters. In particular, it is difficult to obtain the sticking strength accurately.

Through the above simulation results, we verified the effectiveness of our numerical model. We also verified that the relationship between the frequency of the flexible modes and that of the stick-slip motion is important in determining the attitude stability. In the next section, this relationship is investigated in the frequency domain.

\subsection{Sensitivity Analysis}

The disturbance caused by the stick-slip motion was formulated as $-K_{p d l} \Delta q_{f l x}$ in Eq. (7). Now we replace it with the notation $d$ to simplify the description and derive a sensitivity function from $d$ to the attitude angle $q_{\text {att }}$. In this derivation, the effects of feedback attitude control are also considered. The number of dimensions of $d$ is the same as the number of flexible modes, but now the most significant element for ADEOS, which is an element acting directly on the first flexible mode, is considered. Frequency properties of the sensitivity function for each axis are plotted in Fig. 10. The properties depend on the rotational angle of the solar array, so two cases are calculated: $215^{\circ}$ corresponds to the conditions of Figs. 6 and 7 (sunlight) and $300^{\circ}$ corresponds to the condition of Figs. 8 and 9 

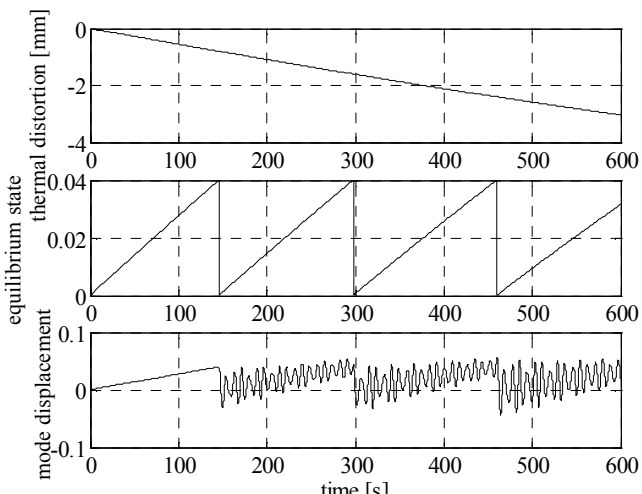

(a) solar array dynamics

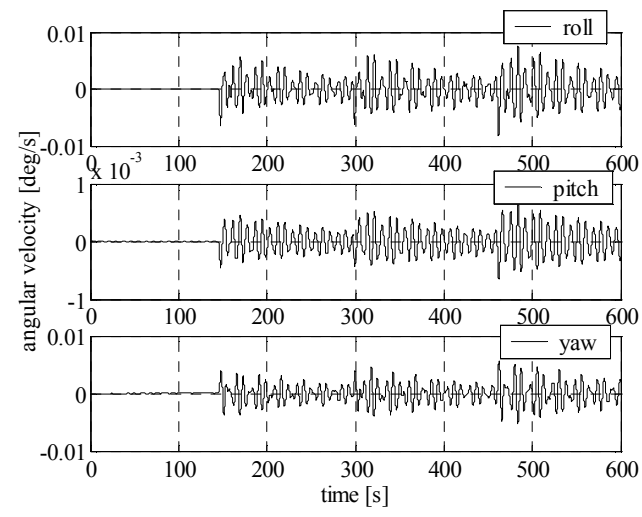

(b) attitude dynamics

Fig. 6 Simulation results (ADEOS, sunlight)

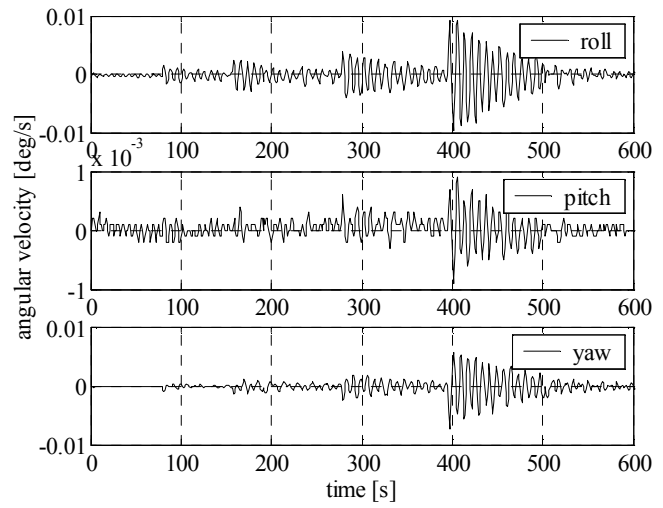

Fig. 7 Telemetry data (ADEOS, sunlight)

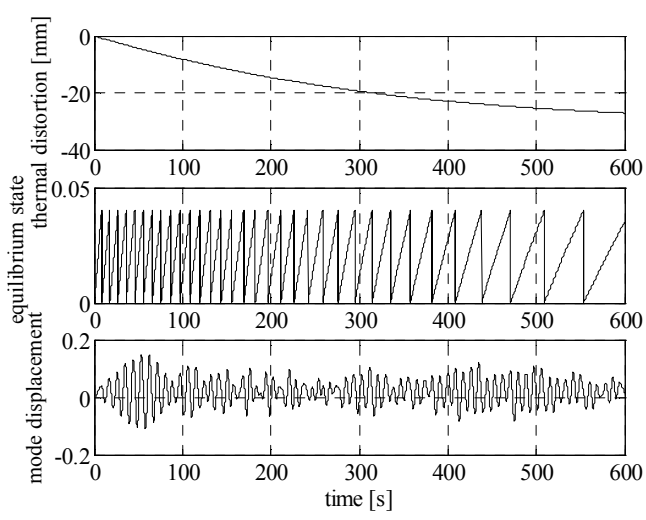

(a) solar array dynamics

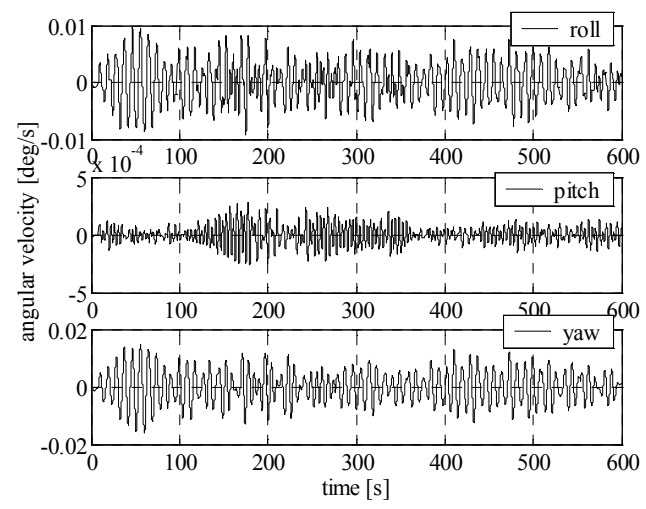

(b) attitude dynamics

Fig. 8 Simulation results (ADEOS, eclipse)

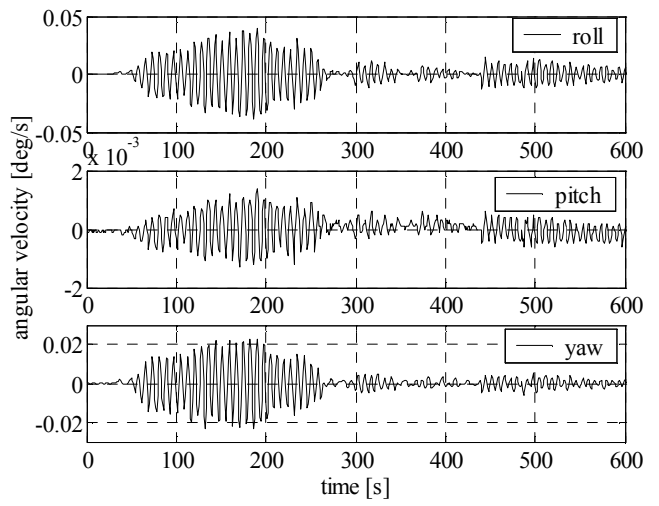

Fig. 9 Telemetry data (ADEOS, eclipse)

(eclipse). There are some peaks from 0.1 to $0.3 \mathrm{~Hz}$, and the peaks of the roll and yaw axes are higher than those of the pitch axis. This is consistent with the time domain analysis in Figs. 6 and 8, where the amplitude of the roll and yaw angular velocities in eclipse are transitional and larger than other cases. The stick-slip frequency in sunlight is $0.007 \mathrm{~Hz}$ and that in eclipse is from 0.1 to $0.025 \mathrm{~Hz}$. Therefore, the stick-slip frequency in eclipse passes through the sensitive frequency.

\section{Measures against Stick-Slip}

The sensitivity at the stick-slip fundamental frequency should be reduced in order to improve the attitude stability. In particular, we can shape the gain plot of the sensitivity function by adjusting the structural design of the blanket and the extension mast and by synthesizing the attitude control parameters. Of course, it is also effective to modify the 

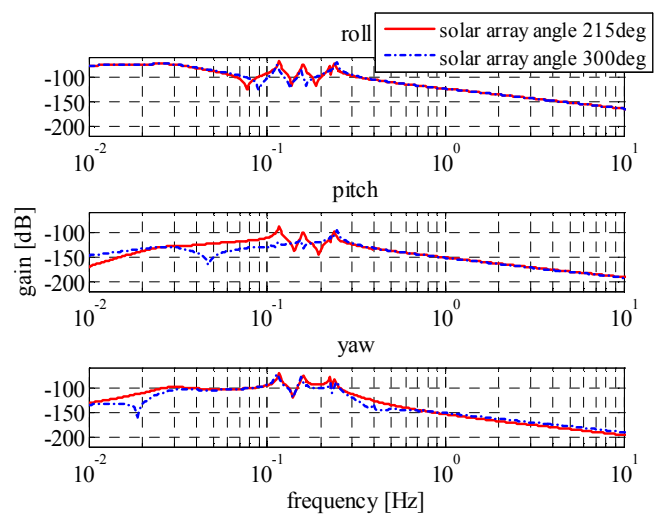

Fig. 10 Sensitivity to stick-slip (ADEOS)

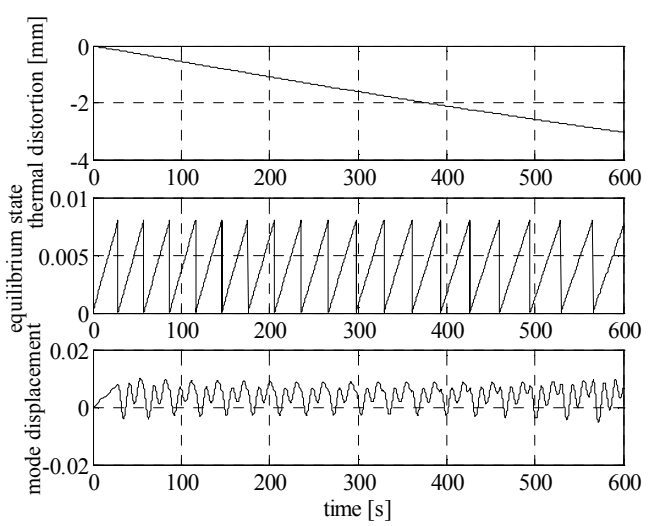

(a) solar array dynamics

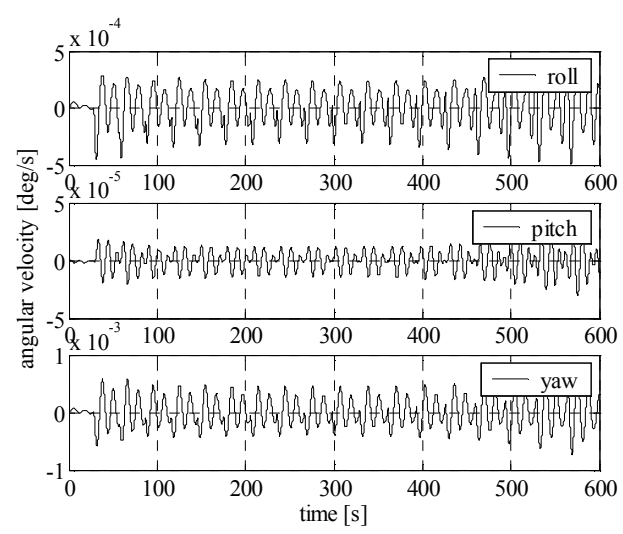

(b) attitude dynamics

Fig. 12 Simulation results (ADEOS-II, sunlight)
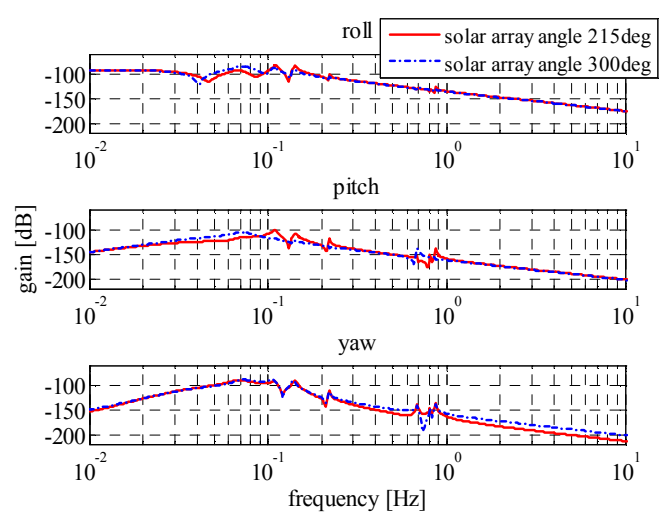

Fig. 11 Sensitivity to stick-slip (ADEOS-II)

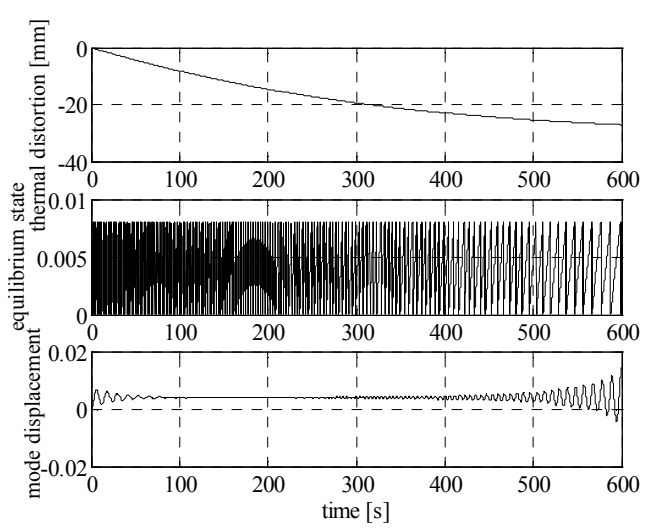

(a) solar array dynamics

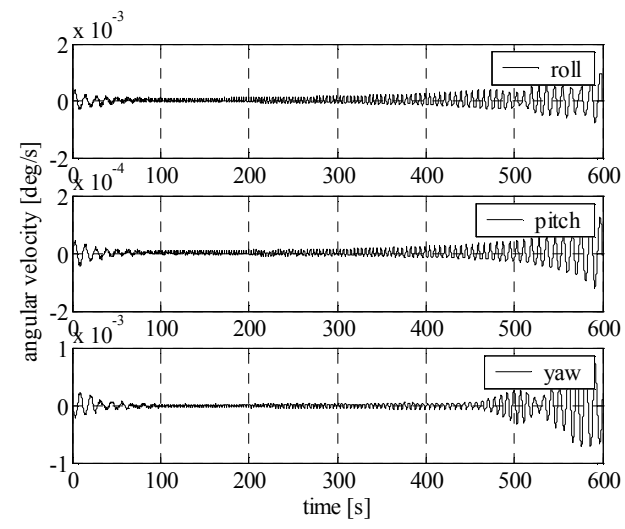

(b) attitude dynamics

Fig. 13 Simulation results (ADEOS-II, eclipse) TCM properties to reduce the sticking strength. If the sticking strength is reduced, the stick-slip cycle becomes shorter and the magnitude of each disturbance is reduced even when stick-slip motion does occur.

A lot of measures against stick-slip motion were tested when ADEOS-II was designed. The frequency properties of the sensitivity function of ADEOS-II are shown in Fig. 11. The overall gain level is lower than that of ADEOS. In addition, the TCM sticking strength of ADEOS-II was designed to be less than $1 / 5$ of that of ADEOS. If we assume that the thermal distortion is the same as in Figs. 6 and 8, the attitude dynamics of ADEOS-II is numerically estimated as Figs. 12 and 13. Figure 12(a) shows that the fundamental frequency of the stick-slip motion in the daytime is about $0.035 \mathrm{~Hz}$. This is close to the sensitive frequency from 0.07 to $0.2 \mathrm{~Hz}$ in Fig. 11. Figure 13(a) shows that the fundamental frequency of the stick-slip motion in eclipse varies from 0.55 to $0.11 \mathrm{~Hz}$. Therefore, it 
passes through the most sensitive frequency at a time between 500 and 600 s. Even for such conditions, the amplitude of the attitude angular velocity is less than $1 / 10$ of that for ADEOS because both the disturbance amplitude and the sensitivity are reduced.

Numerical simulation results for the worst condition, that is, a stick-slip frequency of $0.08 \mathrm{~Hz}$, are shown in Fig. 14. Even in this condition, the attitude angular velocity did not diverge and the required attitude specifications were satisfied.

\section{Conclusion}

We introduced a numerical model to simulate the attitude dynamics of the stick-slip motion of a flexible solar array with a tension control mechanism. In this model, the attitude dynamics with a flexible appendage is formulated as a general constrained mode model, and coupling with the stick-slip motion is formulated as the transition of the equilibrium states of modal displacements. A sensitivity function of the attitude against the stick-slip motion was also derived. The effectiveness of this method was verified by comparing simulation results with actual telemetry data for ADEOS. This method was also applied to ADEOS-II, and the robustness of ADEOS-II against stick-slip motion was verified.

\section{References}

(1) C. L. Foster, M. L. Tinker, G. S. Nurre, W. A. Till, The Solar Array-Induced Disturbance of the Hubble Space Telescope Pointing System, NASA Technical Report 3556 (1996), pp. 1-25, NASA.

(2) John D. Johnston, Earl A. Thornton, Thermally Induced Attitude Dynamics of a Spacecraft with a Flexible Appendage, Journal of Guidance, Control, and Dynamics, Vol.21, No.4 (1998), pp. 581-587.

(3) Earl A. Thornton, Yool A. Kim, Thermally Induced Bending Vibrations of a Flexible Rolled-Up Solar Array, Journal of Spacecraft and Rockets, Vol.30, No.4 (1993), pp. 438-448.

(4) Earl A. Thornton, Gregory P. Chini, David W. Gulick, Thermally Induced Vibrations of a Self-Shadowed Split-Blanket Solar Array, Journal of Spacecraft and Rockets, Vol.32, No.2 (1995), pp. 302-311.

(5) John D. Graham, Solar Induced Bending Vibrations of a Flexible Member, AIAA Journal, Vol.8, No.11 (1970), pp. 2031-2036.

(6) Harold P. Frisch, Thermally Induced Response of Flexible Structures: A Method for Analysis, Journal f Guidance and Control, Vol.3, No.1 (1980), pp.92-94. 